\title{
CRISIS ECONÓMICA DEL ECUADOR: UNA MIRADA AL SECTOR MICROEMPRESARIAL POST COVID-19
}

AUTORES: Josselyn Geovanna Vera Ortiz ${ }^{1}$

Axel Xavier Vera Barzola ${ }^{2}$

María Leonor Parrales Poveda ${ }^{3}$

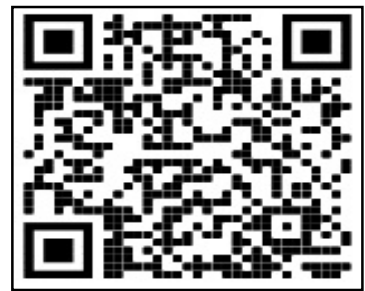

DIRECCIÓN PARA CORRESPONDENCIA: vera-josselyn0843@unesum.edu.ec

Fecha de recepción: 24/09/2020

Fecha de aceptación: 26/11/2020

\section{RESUMEN}

Esta investigación plantea analizar la incidencia en el sector micro empresarial de la crisis económica y sanitaria del Ecuador, desde su Producto Interno Bruto (PIB), el desempleo y aumento gradual de la pobreza. Las ventas por una drástica disminución de la demanda generalizada durante el confinamiento, aunado a la falta de recursos, provocó que las arcas fiscales tengan menores ingresos, el consumo disminuya, así como los ingresos y liquidez. Se realizó una revisión cronológica documental, bibliográfica y cualitativa, que permitió examinar los efectos de la pandemia del Covid-19 en este sector que mueve el $90 \%$ de la economía nacional. Los resultados evidencian que las cifras de la crisis económica se han acentuado en los meses del estado de excepción. La economía decreció un -2,4\% con respecto al periodo del 2019. Desde el sector micro empresarial y políticas de gobierno no se han encontrado soluciones concretas para atenuar los problemas de recesión. Sin embrago, la microempresa se ha convertido en un medio para potenciar el crecimiento socioeconómico del país.

PALABRAS CLAVE: Crecimiento socioeconómico; microempresa; pobreza; recesión.

\section{ECONOMIC CRISIS IN ECUADOR: A LOOK AT THE POST COVID-19 MICROENTERPRISE SECTOR}

\begin{abstract}
This research proposes to analyze the incidence in the micro-business sector of the economic and health crisis of Ecuador from its Gross Domestic Product (GDP), unemployment and gradual increase in poverty. Sales due to a drastic decrease in generalized demand during the Confinement, coupled with the lack of resources, caused the fiscal coffers to have lower income, consumption to decrease, as well as income and liquidity. A chronological documentary, bibliographic and qualitative review was carried out, which allowed us to examine the effects of

\footnotetext{
${ }^{1}$ Universidad Estatal Del Sur De Manabí, Jipijapa, Ecuador. E-mail: vera-josselyn0843@unesum.edu.ec

${ }^{2}$ Universidad Estatal Del Sur De Manabí, Jipijapa, Ecuador. E-mail: vera-axel1814@unesum.edu.ec

${ }^{3}$ Universidad Estatal Del Sur De Manabí, Jipijapa, Ecuador. E-mail: maria.parrales@unesum.edu.ec
} 
Josselyn Geovanna Vera Ortiz, Axel Xavier Vera Barzola, María Leonor Parrales Poveda

the Covid-19 pandemic in this sector that moves $90 \%$ of the national economy. The results show that the figures of the economic crisis have been accentuated in the months of the state of emergency. The economy decreased by $-2.4 \%$ compared to the period of 2019 . From the microbusiness sector and government policies, no concrete solutions have been found to mitigate the problems of recession. However, microenterprises have become a means to boost the country's socioeconomic growth.

KEYWORDS: Socio-economic growth; micro-business; poverty; recession.

\section{INTRODUCCIÓN}

Es evidente que cuando la crisis en un país se encuentra en precipitación, las exportaciones e importaciones, el gasto público, las inversiones y el consumo privado decrecen en consecuencia con la producción de la nación. El Producto Interno Bruto (PIB) ha entrado en un proceso de declive en el trascurso de la crisis económica, sanitaria y social que se está viviendo en el Ecuador, este decayó -7,4\% ubicándose en el cuarto puesto, por debajo de México, Brasil y Perú.

El difícil momento que vive el Ecuador, tiene su génesis, desde que sus gobiernos dejaron de ahorrar e incrementaron el gasto público, producto de esto aumentó el déficit fiscal y la deuda pública, aquella que ha tenido un aumento inadmisible. Actualmente esta deuda es de 59.250 millones de dólares, se ha incrementado en 10 mil millones en un año, bordeando ya los 60 mil millones de dólares. En el transcurso de la pandemia del año 2020, el financiamiento externo llegó a 1.800 millones de dólares; no obstante, las medidas aplicadas por parte del gobierno, no han sido estratégicamente las adecuadas. Para crecer económicamente no basta con tan solo pre vender el petróleo con el riesgo de la caída de su precio, empeñar el oro del estado o utilizar al Banco Central como prestamista del gobierno, cuando no hay ni liquidez para pagar las deudas internas, estas estrategias no han servido para tener la estabilidad económica esperada.

Al respecto, el desarrollo económico del Ecuador en menos de un año ha presentado interrupciones progresivas. En el 2019 con el paro de octubre a causa de las medidas económicas del gobierno de Lenín Moreno, el sector productivo perdió USD 120 millones diarios en venta para el sector micro empresarial, ocasionando también un frenazo productivo en este sector según datos del Comité Empresarial Ecuatoriano (CEE), siendo afectados con pérdidas por un total de USD 720 millones. Este 2020, con la pandemia del Covid-19, se avizoran cambios para los ecuatorianos y la familia micro empresarial.

En tal sentido, las microempresas al ser pequeñas industrias, con cimientos frágiles, que reportan flujos de entrada mínimos, quedan expuestas a un mayor riesgo de liquidez frente a la emergencia sanitaria por el covid-19 que ha ocasionado mayores estragos en ella, como debilitamiento de su estructura, que acompañada con la crisis económica que ya pesaba en Ecuador, ha dejado como resultado un incremento en el desempleo, pobreza y baja producción a nivel nacional, claro está, que si no hay circulante, las empresas producen menos debido a que la demanda se restringe porque no hay dinero para satisfacer las necesidades básicas y sociales.

Sin duda, debido a la crisis económica que vive el Ecuador, es idílico creer que las microempresas no se encuentran perjudicadas, por tal motivo, el objetivo de este trabajo es analizar la incidencia en el sector micro empresarial de la crisis económica y sanitaria del Ecuador, desde el enfoque de la producción, el desempleo y aumento gradual de la pobreza.

2 UNESUM-Ciencias. Publicación cuatrimestral. Vol. 4, No. 4 (Septiembre-Diciembre), Año 2020. 
El artículo inicia detallando la crisis del Ecuador que se ha tornado preocupante y sigue en un decrecimiento gradual, en consecuencia se realiza un estudio del indicador del PIB y las variables del desempleo, pobreza, producción así como a la microempresa; para un resultado efectivo se realiza una revisión documental a través de los métodos bibliográfico y cualitativo, se utilizan materiales audiovisuales con el fin de realizar una búsqueda rigurosa que ayude con la construcción del tema en desarrollo y poder así analizar los efectos de la crisis y la pandemia del Covid-19 en el objeto de estudio. Se aplica una investigación tipo exploratorio-documental, a través del método descriptivo y cualitativo se recolectó información valiosa desde materiales impresos, digitales y audiovisuales como procedimientos de validación del contenido de trabajo.

\section{DESARROLLO}

La economía ecuatoriana se encuentra en una situación de inestabilidad, con altas tasas de desempleo, incremento en su índice de pobreza y una baja producción, que se torna preocupante. La crisis fiscal del 2019, sumándose el escenario de este año que incluye la baja de los precios del crudo, los efectos de casi seis meses de confinamiento, paralización parcial de buena parte de las actividades productivas y comerciales, y reducción de la demanda de productos ecuatorianos en el exterior, muestran un panorama complejo para el sector empresarial, especialmente el de la microempresa. En una economía la empresa es la unidad básica de la organización productiva. Penrose (como se citó en Pomar, Rendón y Hernández, 2006) menciona:

La mayor parte de la actividad económica que incluye la producción, el consumo y la distribución está canalizada por medio de las empresas. La empresa es así "una institución compleja que incide en la vida social y económica de varias maneras, que comprende actividades numerosas y diversas, que toma un amplio número de decisiones significativas. En economía, la empresa tradicionalmente ha sido estudiada bajo el enfoque de "Teoría de la empresa", el equilibrio de la empresa es el volumen de producción para un producto dado (o un conjunto de productos). El crecimiento de las empresas es un resultado del aumento en la producción de artículos dados y la dimensión óptima es el punto que corresponde al mínimo de curva de costos medios de esos productos (1962). En todo caso es importante señalar que no existe dentro de la teoría económica un enfoque particular y específico para la empresa pequeña y mucho menos para la de tamaño micro (Pomar et al, 2006).

Al pasar por una crisis, se evidencian pérdidas, entre el 16 de marzo y el 24 de mayo del 2020; por un lado, el sector productivo en ventas llega a USD 14.101 millones, según el Ministerio de la Producción. El confinamiento paralizó cerca del 70\% de las actividades económicas. El área comercial es la más afectada, con pérdidas que superan los USD 7.600 millones; le siguen las ramas de servicios, manufactura, agricultura y otros. En una depuración de datos del Servicio de Rentas Internas (SRI) sobre las ventas internas del sector privado, en marzo y abril de este año, tres de los segmentos más impactados por la crisis fueron la venta de vehículos, actividades de agencias de viajes y actividades de restaurantes, datos analizados con los Valores Agregados Bruto (VAB) (Tabla 1). 
El costo humano y social de la pandemia por covid-19 es incalculable, pero el económico ya tiene un valor y es cada vez más alto, una variable analizada es el PIB que siguió experimentando reducciones, desde el periodo del 2019 que tuvo un decrecimiento de -0,1\%, estas reducciones se siguen evidenciando en el 2020. En el ámbito internacional, la economía ecuatoriana se ha visto afectada por una caída significativa en los precios de su principal producto de exportación, el petróleo crudo, y un menor dinamismo en la demanda externa de productos no petroleros, resultado de la contracción económica de China, Estados Unidos y la Unión Europea, por las medidas de confinamiento y aislamiento ante el Covid-19 (Banco Central del Ecuador, 2020).

Tabla 1. Cuadro comparativo de la evolución anual del VAB sectorial

\begin{tabular}{|c|c|c|}
\hline Evolución Sectorial del VAB & Año 2019 & Año 2020 \\
\hline $\begin{array}{l}\text { Actividades profesionales, técnicas } \\
\text { y administrativas }\end{array}$ & Creció un 2,9\% & $\begin{array}{l}\text { Decreció en } 1,3 \% \text { con respecto al primer } \\
\text { trimestre de } 2019 .\end{array}$ \\
\hline Alojamiento y servicios de comida & Creció un 2,3\% & $\begin{array}{l}\text { Decreció en } 4,9 \% \text { respecto al primer } \\
\text { trimestre de } 2019\end{array}$ \\
\hline Correo y Comunicaciones & Creció 1,6\% & $\begin{array}{l}\text { Creció un 2,1\% en el primer trimestre de } \\
2020 .\end{array}$ \\
\hline Transporte & Creció un 1,1\% & $\begin{array}{l}\text { Decreció } 4,0 \% \text { en el primer trimestre de } \\
2020 .\end{array}$ \\
\hline Agricultura & Creció un $0,1 \%$ & $\begin{array}{l}\text { Creció un 1,4\% en el primer trimestre de } \\
2020 .\end{array}$ \\
\hline $\begin{array}{l}\text { Enseñanza, servicios sociales y de } \\
\text { salud }\end{array}$ & Disminuyó un 0,5\% & $\begin{array}{l}\text { Disminuyó en } 4,7 \% \text { en el primer trimestre } \\
\text { de } 2020 .\end{array}$ \\
\hline Comercio & $\begin{array}{l}\text { Variación negativa de } \\
0,9 \%\end{array}$ & $\begin{array}{l}\text { En relación a las pérdidas de los } 22 \text { mil } \\
\text { millones de dólares en ventas, el } 50 \% \text { es } \\
\text { de este sector. }\end{array}$ \\
\hline Administración pública y defensa & $\begin{array}{l}\text { Variación negativa de } \\
4,9 \%\end{array}$ & $\begin{array}{l}\text { Variación negativa de } 5,1 \% \text { en el primer } \\
\text { trimestre de } 2020 \text {. }\end{array}$ \\
\hline Construcción & $\begin{array}{l}\text { Decrecimiento de } \\
5,2 \% \text {. }\end{array}$ & Decrecimiento de 7,1\% \\
\hline Refinación de petróleo & Se redujo en $20,4 \%$ & Se redujo en $6,7 \%$ \\
\hline
\end{tabular}

Fuente: Banco Central del Ecuador: Evolución anual del VAB sectorial año 2019 -2020.

Elaborado: Autores

Según Parrales Poveda (2020) en la conferencia del programa de emprendedores de kawsay tech, el VAB y sus principales factores de contratación se encuentran en decrecimiento, en la costa 8,52\% afectando más a la provincia del Guayas y el sector exportador de El Oro y Los Ríos. En la región sierra un -6,92\% tomando en cuenta a Pichincha y en el Oriente -23,91\% que corresponde a la crisis petrolera que enfrenta la Amazonía.

Por otra parte, las consecuencias de la crisis sanitaria y económica que vive el Ecuador se refleja en la calidad de vida de los ecuatorianos. En ese contexto, desde el 29 de febrero de 2020 cuando en el país se confirmó el primer caso de Covid-19, cuatro de las nueve provincias con mayor

4 UNESUM-Ciencias. Publicación cuatrimestral. Vol. 4, No. 4 (Septiembre-Diciembre), Año 2020. 
índice de pobreza del 2019 (Tabla 2) tienen mayor prevalencia del virus y rebasan el promedio nacional de pobreza que es de $18 \%$ (Tabla 3 ).

Tabla 2. Pobreza en las nueve provincias antes de la pandemia de Covid-19

\begin{tabular}{|c|c|c|c|c|}
\hline \multirow[b]{3}{*}{ Provincias } & \multicolumn{4}{|c|}{ Pobreza } \\
\hline & \multicolumn{2}{|c|}{ Pobreza por ingresos } & \multicolumn{2}{|c|}{$\begin{array}{c}\text { Pobreza extrema por } \\
\text { ingresos }\end{array}$} \\
\hline & No pobre & Pobre & No & $\mathrm{Si}$ \\
\hline País & $81 \%$ & $18,3 \%$ & $94,2 \%$ & $5,8 \%$ \\
\hline Guayaquil & $87,0 \%$ & $13,0 \%$ & $97,1 \%$ & $2,9 \%$ \\
\hline Pichincha & $90,1 \%$ & $9,9 \%$ & $97,2 \%$ & $2,8 \%$ \\
\hline Azuay & $88,2 \%$ & $11,8 \%$ & $97,1 \%$ & $2,9 \%$ \\
\hline Manabí & $77,8 \%$ & $22,2 \%$ & $93,9 \%$ & $6,1 \%$ \\
\hline Los Ríos & $76,8 \%$ & $23,2 \%$ & $93,7 \%$ & $6,3 \%$ \\
\hline El Oro & $87,1 \%$ & $12,9 \%$ & $96,7 \%$ & $3,3 \%$ \\
\hline Santa Elena & $78,7 \%$ & $21,3 \%$ & $94,9 \%$ & $5,1 \%$ \\
\hline Esmeraldas & $65,2 \%$ & $34,8 \%$ & $88,9 \%$ & $11,1 \%$ \\
\hline $\begin{array}{l}\text { Santo Domingo de los } \\
\text { Tsáchilas }\end{array}$ & $85,2 \%$ & $85,2 \%$ & $85,2 \%$ & $85,2 \%$ \\
\hline
\end{tabular}

Fuente: Observatorio Social del Ecuatoriano 2019.

Elaboración: autores

La actual crisis global no afecta a todos por igual; la población empobrecida, los microempresarios y trabajadores autónomos e informales son quienes más han sido afectados.

Tabla 3. Pobreza en las nueve provincias después de la pandemia de Covid-19

\begin{tabular}{|l|c|c|}
\hline \multicolumn{1}{|c|}{ País Provincias } & $\begin{array}{c}\text { Pobreza por } \\
\text { ingresos }\end{array}$ & $\begin{array}{c}\text { Pobreza extrema } \\
\text { por ingresos }\end{array}$ \\
\hline País & $\mathbf{1 8 \%}$ & $\mathbf{6 \%}$ \\
\hline Guayaquil & $13 \%$ & $3 \%$ \\
\hline Pichincha & $10 \%$ & $3 \%$ \\
\hline Azuay & $12 \%$ & $3 \%$ \\
\hline Manabí & $21 \%$ & $6 \%$ \\
\hline Los Ríos & $21 \%$ & $6 \%$ \\
\hline El Oro & $23 \%$ & $3 \%$ \\
\hline Santa Elena & $22 \%$ & $5 \%$ \\
\hline Esmeraldas & $35 \%$ & $11 \%$ \\
\hline $\begin{array}{l}\text { Santo Domingo de } \\
\text { los Tsáchilas }\end{array}$ & $15 \%$ & $3 \%$ \\
\hline
\end{tabular}

Fuente: Observatorio Social del Ecuador marzo - junio 2020.

Elaboración: autores

Esmeraldas registra la situación más grave, casi el doble del promedio nacional es pobre de acuerdo con los ingresos que percibe. Mientras que, en las otras cinco provincias de la costa, se 
Josselyn Geovanna Vera Ortiz, Axel Xavier Vera Barzola, María Leonor Parrales Poveda

registran porcentajes de pobreza por ingresos menores al promedio nacional. Sobre la situación de extrema pobreza, Esmeraldas llama la atención nuevamente, esta afecta al $11 \%$ de su población.

\section{Microempresas}

Cuando se habla de la microempresa se refiere a una forma de producción en menor escala y con características personales o familiares en el área de comercio, producción, o servicios que emplea hasta 10 personas como máximo. Según Flores (2018), la microempresa puede ser operada por una persona natural o una familia, de ingresos relativamente bajos, el propietario ejerce un criterio sobre productos, mercados y precios, constituye una importante fuente de ingresos para la familia, en general comprenden a organizaciones económicas promovidas con criterio de solidaridad creando y fomentando programas de inclusión, acorde a la Ley de Economía Popular y Solidaria.

La microempresa puede convertirse en parte de la estrategia de desarrollo y crecimiento de las regiones, dada su capacidad para generar empleo y riqueza. A partir de ello, el diseño de políticas territoriales ha tenido el objetivo de impactar en estas organizaciones. Sin embargo, las políticas tienden a ser de corto alcance, al no responder a las verdaderas necesidades del sector y con ello no reflejar los resultados implícitamente esperados. Se trata de políticas focalizadas desde las instituciones (reconociendo a la microempresa como estrategia de desarrollo social o económico) y no hacia el sector mismo (Díaz Arreguín, 2010).

Según datos del INEC (2019) las grandes empresas agruparon el $71.97 \%$ de ventas, seguidas de la pequeña empresa con 11.29 \%., mientras, las medianas A y B alcanzaron el 15.80 \% y la microempresa el 0.93 \%. Las provincias con mayor participación son Pichincha, Guayas, Azuay, Manabí y El Oro que alcanzan el 88.71 \%. Entre las cinco concentran el 62.58 \% de empresas a escala nacional. Por sector económico se registran cinco tipos de negocios: agricultura, ganadería, silvicultura y pesca; explotación de minas y canteras; industrias manufactureras; construcción; comercio y las de servicios. Además, las ventas de estas simbolizan cerca de $25.7 \%$ del PIB y sobre $10 \%$ de los ingresos netos totales obtenidos en el país, por lo tanto, representan un componente importante de la economía urbana, dato confirmando según el INEC a partir de 2010 hasta 2017.

\section{Microempresas y su comportamiento post covid-19}

La situación económica del Estado ecuatoriano es preocupante, actualmente tiene índices que decrecen gradualmente, y ahora con la emergencia sanitaria por el Covid-19, perjudicó aún más a las empresas y el empleo, la situación se torna más difícil debido a la falta de circulantes y producción de algunos sectores. En el 2019 hubieron inscritas 9.988 empresas y en el transcurso de este mismo año hasta la actualidad el número de cierre ascendió a 432 canceladas y 1.283 en proceso de liquidación. Las más afectadas, según datos del SRI, son las actividades relacionadas al servicio doméstico de los hogares que cayeron en un $72 \%$; le sigue los hoteles y restaurantes con una reducción del 46\% en sus ventas; la explotación de minas y petróleo con el 32\%; y los servicios de las administraciones públicas y la construcción con el 30\% ( Roa Chejín, 2020;Roura y Garcia, 2020).

6 UNESUM-Ciencias. Publicación cuatrimestral. Vol. 4, No. 4 (Septiembre-Diciembre), Año 2020. 
El sector micro empresarial ha sido afectado indiscutiblemente por el covid-19, trayendo consigo una gran inestabilidad económica y subida de precios, baja productividad y desempleo, cuya tasa cayó casi 27 puntos entre junio del 2019 y junio del 2020, según datos del INEC. "En una crisis, las empresas intentan bajar al mínimo sus costos, incluso proyectando ahorros en gastos futuros de jubilación (Silva, 2020).

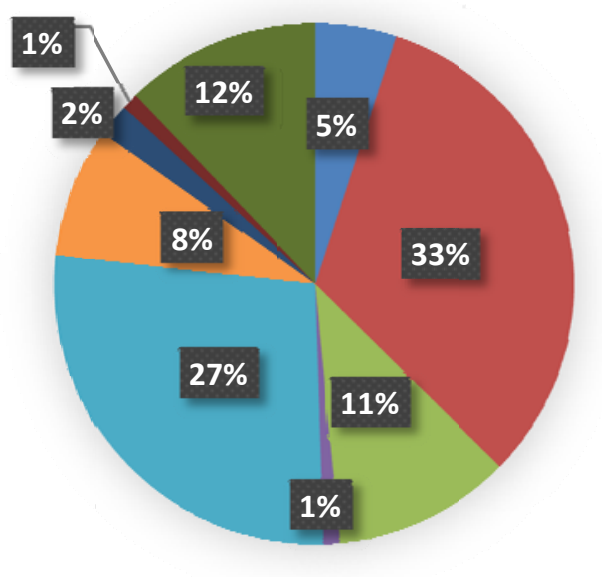

$$
\begin{aligned}
& \text { Liquidación empresas } \\
& \text { Despido intempestivo } \\
& \text { Renuncia voluntaria } \\
& \text { Supresión partidas } \\
& \square \text { Terminación contrato } \\
& \text { Fue mal en el negocio } \\
& \square \text { Terminó ciclo agrícola } \\
& \square \text { Se jubiló o pensionaron } \\
& \square \text { Otro }
\end{aligned}
$$

Figura 1. Causas del desempleo en Ecuador

Fuente: Universidad Central del Ecuador: Causas del desempleo Elaboración: autores

En medio de la emergencia sanitaria provocada por el covid-19, la tasa de desempleo en Ecuador sigue en aumento, es considerada la más alta que se ha registrado desde el 2007 y refleja el fuerte impacto que ha tenido la pandemia y la cuarentena. El incremento fue de 9,5 \% frente a diciembre del 2019, cuando estuvo en 3,8 \%. La principal causa del desempleo, ha sido por despidos intempestivos, mientras que la segunda causa fue la terminación de contratos. Por otro lado, la liquidación de empresas apenas alcanza el 5\% como causa del desempleo (Beltrán, 2020).

\section{Resiliencia e Innovación microempresarial}

En este momento, la comunicación digital se ha convertido para las microempresas en una herramienta esencial, estamos en la era del e-commerce, que ha permitido que las empresas se adapten a nuevas metodologías de comercialización, en donde el comercio directo ha sido desplazado por el comercio electrónico, a través de las redes sociales y medios digitales, logrando de esta manera optimizar sus ventas.

La pandemia del covid-19 ha provocado que muchas empresas cambien sus canales de distribución, de promoción y comunicación, transportándose así a la era de la información, haciendo uso de mensajería, chats, plataformas y redes sociales, siendo esta una forma de adaptarse a los cambios producidos. Las microempresas en el transcurso de estos seis meses de confinamiento, esto es desde el 29 de febrero hasta fines de agosto, han caído en un desequilibrio económico alarmante, cerrando locales por la falta de liquidez para pagar sus deudas, por tal 
Josselyn Geovanna Vera Ortiz, Axel Xavier Vera Barzola, María Leonor Parrales Poveda

razón, han optado por crear páginas web para vender sus productos con mayor facilidad y de esta manera interactuar con el cliente, quien también ha tenido que aprender a confiar en los medios electrónicos para dotarse de lo necesario.

\section{Apoyo del Estado ecuatoriano}

Ecuador recibió un préstamo por 260 millones de dólares que fue aprobado por el Directorio Ejecutivo del Banco Mundial (BM) para la Corporación Financiera Nacional (CFN)para apoyar asía las Mipymes apoyando así su reactivación y recuperación económica, tras las grandes pérdidas que dejó el Covid-19.El Gobierno Nacional creó el Programa Reactívate Ecuador con la finalidad de aliviar los impactos de la pandemia que han tenido que asumir las Mipymes.

Como una de las secuelas de las medidas sanitarias estrictas de la pandemia del 2020 la rutina de compra y venta sufrieron cambios, y de igual forma el hábito del consumo de los clientes y proveedores, lo que impulso a la innovación de muchos microempresarios, los recursos económicos resultado del préstamo otorgado fueron utilizados como capital de trabajo, pago de nóminas, y para pagar los costos operativos, por otra parte, para la mayoría del sector micro empresarial fue la oportunidad para hacer su salto al e-commerce, logrando llegar a nuevos clientes potenciales y satisfacer a sus reales. La presión que han tenido estas pequeñas industrias a desembocando en una evolución y el deseo de incursionar en las nuevas tecnologías para actualizarse de manera permanente.

A pesar del apoyo gubernamental, estas siguen teniendo retos: la falta de confianza, problemas técnicos, la deficiente relación directa con los consumidores y proveedores, y la competencia, se ha convertido en dificultades para tener resultados favorables; desde cualquier perspectiva hoy en día las desventajas están superando a las ventajas, las políticas públicas, siguen siendo aún muy débiles en su asignación económica y focalización. Como se puede inferir, la crisis económica ha ganado espacio a nivel nacional, baja producción, niveles altos de desempleo y una creciente pobreza avizoran un panorama gris para el sector objeto de estudio.

\section{RESULTADOS}

La población objeto de estudio ha sido la microempresa y cómo la crisis han impactado en su desarrollo. El material bibliográfico ha sido tomado de fuentes como revistas Vistazo, Primicias, Perspectiva y de Sistema de información científica; diarios escritos de cobertura nacional y confiabilidad, como El Comercio, El Universo y El Mercurio; materiales audiovisuales como el canal de Noticias Ecuavisa, Ponencias en congresos y eventos virtuales; sitios web confiables como el Banco Central del Ecuador, La Cámara Marítima del Ecuador, El Ministerio de Economía y Finanzas, El Ministerio del Trabajo, El Instituto Nacional de Estadísticas y Censo del Ecuador, entre otros, con el fin de fundamentar el tema en desarrollo con información confiable y fidedigna.

La revisión bibliográfica que se utilizó en este estudio sirvió para identificar:

i. Las cifras de la crisis económica siguen siendo negativas, existe un decrecimiento de 2,4\% con respecto al período de 2019, según los datos de las Cuentas Nacionales

8 UNESUM-Ciencias. Publicación cuatrimestral. Vol. 4, No. 4 (Septiembre-Diciembre), Año 2020. 
publicadas por el BCE. Los expertos consideran que la recesión económica que enfrenta el país solo puede ser comparada con la crisis financiera y social de hace 20 años; los números reflejan un panorama negativo tanto para las finanzas públicas y privadas durante los 6 meses aproximadamente de vigencia del estado de excepción.

ii. La caída de las ventas, por una drástica disminución de la demanda generalizada durante los meses de confinamiento y la falta de recursos provocó que las arcas fiscales tengan menores ingresos por impuestos; la recaudación tributaria de enero a julio del 2019 fue de unos 8.100 millones de dólares mientras que en el mismo periodo del 2020 con la propagación del coronavirus bajó cerca de 6.800 millones de dólares (Aizprúa, 2020).

iii. Las investigaciones de Coba (2020), han respondido la pregunta ¿Por qué las microempresas están en proceso de liquidación?. "Las microempresas son negocios que reportan flujos de entrada mínimos, quedando expuestas a un mayor riesgo de iliquidez. La mitad de estos negocios solo resiste 17 días sin recibir efectivo, las Pymes, pueden aguantar 31 y 32 días, respectivamente”. En el trascurso de los seis meses que el Estado ecuatorianos ha estado en confinamiento ya han pasado 180 días aproximadamente, número que evidencia las microempresas canceladas y en proceso de liquidación.

El Ecuador, es un país pequeño, con limitada capacidad de ahorros, sin duda alguna era el país que más saldría afectado, sus bases económicas, sociales y empresariales no estaban fortalecidas, por lo tanto, se convertiría en una nación de alto riesgo país, el cual llegó a 6.063 puntos, presentando hasta el 10 de septiembre un descenso que llegó a 952 puntos por debajo de Venezuela y Argentina, debido a la renegociación de los bonos de deuda externa. Desde el mes de octubre de 2019, por las medidas económicas y el decreto 893 impuesto por el ejecutivo, se generalizaron protestas en todo el país y con esto un debilitamiento más en su economía.

Según el Ministerio de Finanzas, el programa Reactívate Ecuador hasta el 01 de agosto otorgó crédito a 2.200 MiPymes, al desglosar esta cantidad en porcentaje se observa que el $8 \%$ de recursos a microempresas, el $54 \%$ a las pequeñas medianas y el $38 \%$ a las medianas. Las ciudades con mayor beneficio del crédito fueron: Quito 36\%, Guayaquil 26\%, Santo Domingo, $65 \%$, Ambato 5\%, Cuenca 4\%, y el resto del país 23\%. Este programa tuvo el objetivo de minimizar los estragos económicos y minorar la situación compleja producto de la pandemia (ACR, 2020).

La provincia del Guayas se ha convertido en un "Modelo Nacional” de recuperación económica, apoyará a la microempresa eliminando la tasa de habilitación para funcionamiento de establecimientos hasta el año 2021, para todo comercio (tiendas, gimnasios, consultorios y hoteles) asentados en la ciudad; además, realizarán un descuento del $50 \%$ en el pago de impuestos prediales y otras tasas a las nuevas industrias que se asienten en la urbe.

Menciona Beltrán (2020), que el coronavirus ha golpeado fuerte el mercado laboral ecuatoriano, el número de desempleados sumando los 36.000 que ya estaban sin empleo antes de la pandemia, llegan alrededor de 1,8 millones entre junio del 2019 y junio del 2020 a nivel nacional y otros 1,6 
Josselyn Geovanna Vera Ortiz, Axel Xavier Vera Barzola, María Leonor Parrales Poveda

millones de trabajadores no percibieron un solo dólar de ingreso en mayo. La tasa de desempleo a nivel nacional alcanzó un 13,3 \% en mayo y junio del 2020, según cifras del INEC.

Según el presidente de la República del Ecuador, Lenín Moreno, la Ley Humanitaria ha empezado a rendir sus frutos, refiriéndose a los indicadores del empleo, ya que ha logrado salvar 56000 empleos, a través de la reducción de la jornada de trabajo. Además, han facilitado 104000 nuevos empleos pese a la pandemia. De acuerdo con datos facilitados por el Ministerio de Trabajo, entre el 22 de junio y el 2 de septiembre se han reportado 56588 contratos de trabajo vigentes con reducción de jornada. Pese a estos nuevos contratos, el saldo sigue siendo negativo. Según los propios datos del Ministerio de Trabajo si bien se crearon entre enero y septiembre 254 357 empleos y hubo 538108 actas de finiquito. (Círculo de Estudios Latinoamericana, 2020)

Uno de cada cuatro ecuatorianos son pobres, y luego de la pandemia serán más, (2020) las estimaciones del Banco Mundial (BM)menciona que, hasta diciembre de este año, el índice de pobreza estará en un 35\% de la población y la extrema pobreza se ha situado en el $21 \%$ en mayo y junio del 2020, como efecto inmediato de la pandemia, Pobreza por ingreso familiar per cápita USD 84.79 mensuales o menos, mientras que la extrema pobreza por ingresos quienes perciben un per cápita USD 47.70 mensuales.

La Cámara de Industrias y Producción (CIP), está elaborando una propuesta, con 17 pilares, para darle sostenibilidad a las empresas en la época post Covid-19. El inicio de la recuperación podría darse en alrededor de 12 a 18 meses. La CIP, que representa a 55 sectores de la economía o el 33\% del PIB, está trabajando de la mano de la Academia e instituciones, como la Organización Internacional del Trabajo (OIT), con el objetivo concretar una agenda de competitividad, el impulso al consumo nacional y el proyecto de Empresas Sostenibles (Cámara Marítima del Ecuador , 2020).

\section{DISCUSIÓN}

El Ecuador es el país más pequeño de Sur América Continental, exporta productos de bajo valor agregado al igual que sus países vecinos del Norte (Colombia y Venezuela). El $90 \%$ de sus exportaciones corresponde a petróleo crudo, banano y mariscos, a pesar de tener un buen margen de exportación la variación porcentual del PIB no ha sido tan significativa en estos últimos años.

La producción de las personas, empresas y gobierno en el Ecuador han tenido un descenso preocupante en los meses de la pandemia provocada por el Covid-19, esta producción llega a ser medible a través del indicador del PIB, aquel que ha pasado por un retroceso económico violento producto de la crisis económica y sanitaria que vive el Estado ecuatoriano, crisis que ha traído consigo problemas que se encuentran interconectados y repercuten en la diminución de la producción, el desempleo y la pobreza; las empresas canalizan muchas actividad en función del bienestar social y económico, coincidiendo con el criterio de Penrose, las empresas llegan a ser una unidad básica de la economía de una región e inciden en diversas acciones productivas.

Como consecuencia del frenazo productivo, las inversiones, el gasto público del estado, las exportaciones e importaciones han disminuido, dicho en otras palabras, la Renta Nacional del Ecuador se encuentra en una fase de recesión y en efecto, el PIB per cápita de cada ciudadano es

10 UNESUM-Ciencias. Publicación cuatrimestral. Vol. 4, No. 4 (Septiembre-Diciembre), Año 2020. 
negativo. Es evidente que no estamos económicamente estables y en comparación con otros países latinoamericanos estamos en el cuarto lugar en decrecimiento con un -7,4\% por debajo de México, Brasil y Perú, porcentaje que podemos comprobar con las proyecciones del BCE.

Si una economía crece significativamente produce más, y las empresas llegan a aumentar su producción obteniendo más ventas y mayores ingresos, aquellos que se dividen en todos los recursos humanos involucrados en la producción, es decir que cuando existe mayores ingresos en la nación los ciudadanos pueden optar por una mejor calidad de vida, una realidad que no se puede apreciar en la actualidad en el Ecuador; el sector productivo ya ha presentado perdidas como resultado del confinamiento que paralizó cerca del 70\% las actividades productivas, con un decrecimiento económico las empresas y microempresas sienten más miedo a invertir y las familias no tienen seguridad económica ni social, por lo tanto, dicho decrecimiento también impacta al empleo, las microempresas y a la sociedad, con menos producción y poca inversión, no hay plazas de empleo, incuestionablemente las empresas a producir menos se deshacen de su personal.

Según Martínez Pellégrini (2004) subraya que de acuerdo a las teorías del desarrollo endógeno, las microempresas pueden representar uno de los pilares del desarrollo al ser una opción de generación de empleo e ingreso para la población; estas mismas teorías centran la atención en la importancia de redes y sistemas de microempresas locales como una alternativa viable en el contexto actual de internalización de las economías.

Manteniendo la línea de pensamiento de Martínez Pellégrini, las microempresas se han convertido en la base económica de la sociedad, pero con pocos niveles y posibilidades de supervivencia, a pesar de ocupar un lugar de importancia para el PIB. En Ecuador las microempresas particularmente se dedican a los sectores de comercio, gastronomía, construcción, industrias (mecánicas, ebanistas confección y agroindustrias), antes de la pandemia y con tan solo la crisis económica que enraizaba el Ecuador, estas siempre se habían caracterizado por el empuje que le dan al negocio y el ingenio que manejan para producir con limitados recursos.

En los últimos meses, con el estado de excepción y como resultado del confinamiento, este sector micro empresarial cayó en una situación desfavorable, por falta de recursos y apoyo gubernamental para mermar los impactos económicos que la pandemia del 2020 deja como secuela, aparte de ello, no existe un escenario bancario claro para acceder a créditos y así reactivarse económicamente y salir de la insolvencia que se encuentran, cumplir con las obligaciones tributarias y fiscales.

Por un lado los datos procesados de la CIP hasta agosto de este año indican que las sociedades, personas naturales, y el sector de la economía popular y solidaria tuvieron una caída en ventas de más de 15.500 millones de dólares, aunque la Cámara de Comercio de Quito, destaca que la cifra puede ser mayor, según las cifras de SRI hasta julio, el decrecimiento de ventas es de 20 mil millones y el 93\% de este decrecimiento está en el sector productivo.

El gobierno ni los microempresarios han encontrado soluciones concretas para mitigar los problemas que están pasando y así no cesar en sus funciones, a pesar de que las microempresas 
también se caracterizan por su adaptabilidad y flexibilidad muchas optaron por cerrar y otras se acogieron al nuevo modelo de comercialización del e-commerce y dieron un salto al comercio digital, fomentando así la innovación al entrar este tipo de mercado y realizando sus ventas con distintas metodologías y estrategias, como la famosa feria digital que se realiza en Ecuador, siendo esto parte de sus capacidades de adaptabilidad.

La microempresa en las últimas décadas se ha convertido en un medio para atenuar el desarrollo socioeconómico. Para que el Estado ecuatoriano tenga su reactivación económica e incremente su desarrollo, necesita proporcionar a la población la oportunidad de trabajar, una vez logrado esto, se procede a desarrollar pequeñas empresas, cadenas productivas. Aseverando que el desarrollo solo podrá alcanzarse a través de la promoción de políticas y programas de reducción de la pobreza, fomento del trabajo estable, y generación de empleo.

El empleo, es la forma que tiene un individuo de obtener ingresos a cambio de prestar sus servicios, en Ecuador se estima que de cada diez empleados solo dos tiene un empleo adecuado y ocho están en la desocupación y subempleo, para lograr amortiguar algunas consecuencias de la pandemia el Estado ecuatoriano aprobó la Ley Humanitaria aquella que vista de muchas perspectivas no sirvió de gran apoyo, ni logró cumplir con uno de sus objetivos, proteger los derechos de los empleados y a las microempresas, por otra parte, el aparato productivo también se encuentra debilitado, el mercado laboral deteriorado y como consecuencia de esto1,8 millones perdieron un empleo adecuado entre junio del 2019 y junio 2020, la pandemia como problema global que afecta al Ecuador está vulnerando los derechos individuales y colectivos de los trabajadores.

\section{CONCLUSIONES}

Atendiendo a las consideraciones de este trabajo, se puede concluir que la calidad de vida ha tenido una disminución como causa colateral de la baja producción, la inestabilidad de las empresas y el desempleo; el nivel de pobreza a seguido en aumento, en Ecuador se estima que llegue a $35 \%$ y la pobreza extrema a $21 \%$, estas estimaciones están ligadas a los despidos intempestivos, la terminación de contratos y la liquidación de las empresas, además a la falta de compromiso, de motivación e interés por salir adelante ha provocado que este índice siga creciendo inevitablemente, acentuando la crisis que abruma cada vez más al sector micro empresarial.

\section{REFERENCIAS BIBLIOGRÁFICAS}

ACR. (2020, 01 de agosto). El 4\% Reactivate Ecuador está en Cuenca. Obtenido de El Mercurio.com.ec: https://ww2.elmercurio.com.ec/2020/08/01/solo-4-de-reactivate-ecuador-en-cuenca/

Aizprúa, J. C. (2020, 07 de septiembre ). La cifra negativa de la Crisis Económica. [video].Obtenido de Comunicación Quito Ecuavisa: https://www.youtube.com/watch?v=s6JpQXd-yQA\&t=2659s

Banco Central del Ecuador. (2020, 03 de Junio). El covid-19 pasa factura a la economía ecuatoriana: decrecerá entre 7,3\% y 9,6\% en 2020. Obtenido de bce.fin.ec: https://www.bce.fin.ec/index.php/boletines-de-prensaarchivo/item/1366-el-covid-19-pasa-factura-a-la-economia-ecuatoriana-decrecera-entre-73-y-96-en-2020 
Banco Central del Ecuador. (2020, 30 de Junio). La economía ecuatoriana ha decrecido en el primer trimestre. Obtenido de bce.fin.ec: https:/www.bce.fin.ec/index.php/boletines-de-prensa-archivo/item/1370-laeconom\%C3\%ADa-ecuatoriana-decreci\%C3\%B3-24-en-el-primer-trimestre-de-2020

Banco Central del Ecuador. (2020, 17 de enero). LA ECONOMÍA ECUATORIANA SE RECUPERARÁ 0,7\% DURANTE EL 2020. Obtenido de bce.fin.ec: https://www.bce.fin.ec/index.php/boletines-de-prensaarchivo/item/1348-la-econom\%C3\%ADa-ecuatoriana-se-recuperar\%C3\%A1-07-durante-el2020\#: :text=Para\%20el\%20a\%C3\%B1o\%202020\%2C\%20la,nominal\%20de\%20USD\%20109.667\%20mill ones.

Beltrán, J. (08 de agosto de 2020). La tasa de desempleo del Ecuador de 13,3 \% en junio es la más alta desde el 2007. Obtenido de El Universo.com: https://www.eluniverso.com/noticias/2020/08/08/nota/7935135/desempleo-inec-cifras-pandemia-covid-19

Cámara Marítima del Ecuador. (2020, 28 de agosto). El inicio de la recuperación económica tomaría entre 12 y 18 meses. Obtenido de CAMAE.org: http://www.camae.org/economia/el-inicio-de-la-recuperacion-economicatomaria-entre-12-y-18-meses/

Círculo de Estudios Latinoamericana. (2020, 07 de Septiembre). Ministerio de Trabajo reporta 538108 actas de finquito entre enero y septiembre de 2020. Obtenido de CESLA.com: https:/www.cesla.com/detalle-noticiasde-ecuador.php?Id=15670

Coba, G. (2020, 24 de Abril). Primicias.ec. Obtenido de Crisis económica por Covid-19 reduce cuatro ingresos de Ecuador: https://www.primicias.ec/noticias/economia/crisis-economia-reduce-ingresos-ecuador/

Díaz Arreguín, S. (2010). LA MICROEMPRESA EN EL DESARROLLO. [Artículo científico]. Perspectivas, p. 13.

Flores, J. (2018, 10 de julio). MICROEMPRESA. Obtenido de Derecho Ecuador.com: https://www.derechoecuador.com/microempresa

INEC. (2019, 10 de diciembre). Cerca de 900 mil empresas se registraron en Ecuador en 2018. Obtenido de ecuadorencifras.gob.ec: https://www.ecuadorencifras.gob.ec/cerca-de-900-mil-empresas-se-registraron-enecuador-

en2018/\#: :text=Las\%20grandes\%20empresas\%20agruparon\%20el,y\%20la\%20microempresa\%20el\%200.9 $3 \% 20 \% 25$.

Martínez Pellégrini, S. E. (2004). Necesidades y prioridades de las microempresas y su potencial como arraigadoras de la población mediante generación de empleo e ingreso en México. México: USAID.

Observatorio Social del Ecuador. (2020, 28 de junio). El Covid-19 recorre las desigualdades del Ecuador ¿sobrevivir o quedarse en casa? Obtenido de covid19ecuador.org/: https://www.covid19ecuador.org/post/desigualdades-covid19-ecuador

Pomar Fernández, S., Rendón Trejo, A., y Hernández Mar, R. (2006). La Microempresa. Reconociendo su Importancia. [Artículo científico]. Academia de Ciencias Administrativas AC (ACACIA), 29.

Parrales, M. L. (2020, 18 de agosto). El emprendimiento y las Metodologías Ágilessocioafectivas.[Ponencia].Programa de desarrolladores de emprendimiento. Kawsay Tech.Guayaquil.

Roa Chejín, S. (2020, 14 de Junio). GK. Obtenido de El impacto del covid-19 en la caída del PIB: https://gk.city/2020/06/14/pib-ecuatoriano-caera-6-fmi/

Roura, C., y Garcia, F. (2020, 03 de mayo). ¿El desempleo es la próxima pandemia?. Políticamente Correcto. [video]. Obtenido de youtube: https://www.youtube.com/watch?v=hJINDB2ypao\&t=198s 
Josselyn Geovanna Vera Ortiz, Axel Xavier Vera Barzola, María Leonor Parrales Poveda

Silva, M. V. (2020, 13 de agosto). La pérdida de empleos en Ecuador. Obtenido de ElComercio.com: https://www.elcomercio.com/actualidad/desempleo-empleos-ecuador-afecta-edad.html

14 UNESUM-Ciencias. Publicación cuatrimestral. Vol. 4, No. 4 (Septiembre-Diciembre), Año 2020. 